LA-UR- $93=1438$

Approved for oublic release: distribution is unimited.

\author{
Title:
}

Combinatorics, Geometry, and Mathematical Physics

Author(s):

William $Y$. C. Chen, T-7

James D. Louck, T-7

Submitted to:

DOE Office of Scientific and Technical Information (OSTI)

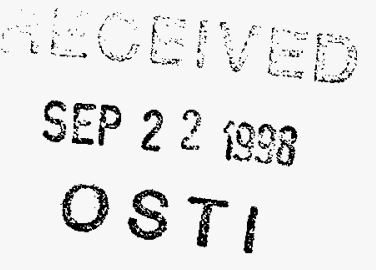

Los Alamos National Laboratory, an affirmative action/equal opportunity employer, is operated by the University of Calitornia for the U.S. Department of Energy under contract W-7405-ENG-36. By acceptance of this articte, the publisher recognizes that the U.S.

Government retains a nonexciusive. royalty-free license to publish or reproduce the published form of this contribution, or to allow others to do so. for U.S. Government purposes. Los Alamos National Laboratory requests that the publisher identify this article as work performed under the auspices of the U.S. Department of Energy. The Los Alamos National Laboratory strongly supports academic freedom and a researcher's right to publish: as an institution, however, the Laboratory does not endorse the viewpoint of a publication or guarantee its technical correctness. 


\section{DISCLAIMER}

This report was prepared as an account of work sponsored by an agency of the United States Government. Neither the United States Government nor any agency thereof, nor any of their employees, makes any warranty, express or implied, or assumes any legal liability or responsibility for the accuracy, completeness, or usefulness of any information, apparatus, product, or process disclosed, or represents that its use would not infringe privately owned rights. Reference herein to any specific commercial product, process, or service by trade name, trademark, manufacturer, or otherwise does not necessarily constitute or imply its endorsement, recommendation, or favoring by the United States Government or any agency thereof. The views and opinions of authors expressed herein do not necessarily state or reflect those of the United States Government or any agency thereof. 


\section{DISCLAIMER}

Portions of this document may be illegible in electronic image products. Images are produced from the best available original document. 


\title{
Combinatorics, Geometry, and Mathematical Physics
}

\author{
William Y. C. Chen* and James D. Louck
}

\begin{abstract}
This is the final report of a three-year, Laboratory-Directed Research and Development (LDRD) project at the Los Alamos National Laboratory (LANL). Combinatorics and geometry have been among the most active areas of mathematics over the past few years because of newly discovered inter-relations between them and their potential for applications. In this project, we set out to identify problems in physics, chemistry, and biology where these methods could impact significantly. In particular, our experience suggested that the areas of unitary symmetry and discrete dynamical systems could be brought more strongly under the purview of combinatorial methods. Unitary symmetry deals with the detailed description of the quantum mechanics of many-particle systems, and discrete dynamical systems with chaotic systems. The depth and complexity of the mathematics in these physical areas of research suggested that not only could significant advances be made in these areas, but also that here would be a fertile feedback of concept and structure to enrich combinatorics itself by setting new directions. During the three years of this project, our goals have been realized beyond expectation, and in this report we set forth these advancements and justify our optimism.
\end{abstract}

\section{Background and Research Objectives}

Unitary symmetry and physical theory. The importance of unitary symmetry in understanding the behavior of complex quantal physical systems is an outgrowth of papers principally by E. Wigner, V. Bargmann, and G. Racah during the 1930s-1950s. These basic papers set the stage for an explosive growth (with hundreds of contributors) in the conceptual foundations and implementations of technique from the 1960s to the present. This has led to fundamental understanding and developments in the areas of molecular, chemical, atomic, nuclear, and particle physics that would not have been otherwise possible. One of the early participants in these activities was J. D. Louck with significant contributions [1]-[3] and a sustained activity in a long series of collaborations with L. C. Biedenharn and others from 1967 to the present, where [3]-[10] are especially relevant to this project. Despite the intense activity in this area, little attempt by physicists and chemists was made to relate the methods to the developments in combinatorics and discrete mathematics, which were occurring simultaneously, nor did the combinatorialists pay much attention to these developments in physical theory.

*Principal Investigator, email: chen@t7.lanl.gov 
The linkage between combinatorics and geometry in the context of this project may be made in the following way:

(i) The geometry arises from the symmetry of phase space and time as embodied in models of complex physical systems, especially in quantum physics, but not exclusively so. The extra richness of structure for quantal systems is a consequence of the Hilbert space used to describe the states of such systems. Symmetry of space-time is implemented through group actions and group representations on such state spaces, leading to a separation of a universal intrinsic geometry of a wide class of physical systems from their particular behavior due to their special circumstance. Such techniques have wide applications to chemistry, physics, and, perhaps, to biology as its understanding becomes based on microscopic law. Many-particle systems are perceived of as being composite systems built-up from simpler fundamental units, and it is here that symmetry has its most powerful impact, leading at the same time to the most intricate and least understood mathematics, that of reducing Kronecker product representations of groups into their elementary, irreducible constituents. The mathematics of representation theory is continuous and discrete (analysis with functions labeled by quantum numbers) with its own geometry and combinatorics, while that of the reduction process is discrete (quantum state labels alone) with its quite different geometry and combinatorics.

(ii) The combinatorics arises, in part, from the study of symmetry in the abstract. Modern combinatorics, for example, encompasses the study of the symmetric group (the group of permutations of collections of identical objects), and the structural relations between a variety of symmetric functions. This is all developed from the viewpoint of special techniques such as bijections and assembly rules for making complex objects from simpler constructs that the proponents of combinatorics have developed and found to have universal appeal. The structure of the special functions of mathematical physics falls under the purview of this approach. The need for symmetric functions in the study of states of physical systems is well-known from the Pauli principle. But the understanding of the symmetry of physical systems goes far beyond this; for example, the ubiquitous general unitary group appears in nearly countless applications. Yet the combinatorics of this group, especially in the discrete arena of its reduction coefficients, has been barely touched upon. The umbral calculus, a modern farreaching theory of formal alphabets and their formal polynomials, based on the concept of double-tableaux, and developed by the Rota school of combinatorialists, bears strongly on this subject, but the definitive account of the relationships has yet to be written. Hints at a deeper structure have emerged from the discovery [4]-[6] of generalized Schur functions and generalized symmetric hypergeometric functions embedded in the 
properties of SU(3) Clebsch-Gordan coefficients, whose structural features are amenable to the interpretations of combinatorics (Publications 1-2).

Fragmentary connections to combinatorics, and complementary to the physics, that emerged from research in unitary symmetry by physicists included: (1) The connection made in [8] between $3 n-j$ coefficients, which are coefficients defined by physicists for the study of complex quantal systems, the theory of binary trees, and the theory of cubic graphs; (2) The formulation [3, 8] of the famous Clebsch-Gordan problem for reducing Kronecker products of irreducible representations of groups to the study of an operator algebra that goes far beyond that of a Lie algebra. There is an associated calculus [9] of dots and arrows associated with this algebra, called the pattern calculus, and it seems almost certain that there is a new body of combinatorics underlying it. (3) The discovery [10] of the fundamental role of the Littlewood-Richardson numbers in resolving a multiplicity problem in the operator theory; these Littlewood-Richardson numbers are basic in combinatorics. (4) The discovery [4] of a beautiful generalization of the Gauss hypergeometric function to many variables. Surprisingly, these objects had also been discovered in statistical analysis and are known there as zonal harmonics. (5) The discovery [5] of a far-reaching generalization of the classical Schur function, a function of fundamental importance in combinatorics.

It was against this background that the collaboration between Chen and Louck was launched in publications 1-4 with the objective of bringing these topics within the framework of combinatorics in the mathematicians sense (Chen is a trained combinatorialist of the MIT Rota School). Much of this combinatorics-related mathematics originating from these unitary symmetry studies in physical theory is still undeveloped, but some of it has now been taken up by mathematicians [12]-[15], primarily because of our efforts in the research carried out under this project.

The following problems were set forth as a realistic collection on which progress could be made, which we repeat exactly from our original proposal:

(1) Structure of Representations. The relationship between double-Gel'fand patterns and double-tableaux for the enumeration of the irreducible representations of the unitary group is well-known. What is not understood, mostly because it has not been studied, is the relationship between the representations constructed through the umbral calculus of Rota, with its important "straightening formula," and the unitary representations constructed by the method of "highest weights," as employed by physicists. Perhaps one can learn from these two methodologies why it is that irreducible representation functions appear to depend for their construction only on the ClebschGordan coefficients of a unitary group of lower order. 
(2) Structure of Reduction Coefficients. In the physicist's language, we refer here to the Clebsch-Gordan and Racah coefficients of a group, these coefficients arising in the reduction of Kronecker products of irreducible representations and tensor operators into irreducibles. This is discrete mathematics of the highest complexity. Indeed, the physicist presents formulas for these quantities of such complication and lack of structure and beauty that the mathematician will pay no attention to them. What a pity, because when the effort is make to bring some order and structure to these objects, one discovers new mathematics of singular quality [4]-[6], much, if not all, of which falls within the purview of modern combinatorics. This source has hardly been tapped! The pay-off for productive interplay between combinatorics and the geometry of symmetry is greatest in this area, one which offers at the same time the greatest challenge for advancement.

(3) Intertwining Function. This function has an important role in the study of both (1) and (2). Numerically, it just counts the number of irreducible representations contained in the Kronecker product of irreducible representations. Properly interpreted as in $[10,11]$, it is an integer-valued function labeled by pairs of ordered partitions and defined over the lattice points of a Möbius space of many dimensions. It is a combinatoric object of great beauty, related in a basic and intrinsic manner to all the other great combinatorial objects of invariant theory [16]. Yet the classification of the structure of the level subspaces (spaces of constant range) of this function has barely been touched upon, despite the fact that these subspaces are the primary object in arriving at a natural classification of the Clebsch-Gordan coefficients for the unitary group.

(4) Generating Functions. The interpretation of generating functions for all kinds of mathematical objects is one of the great triumphs of combinatorics. Such generating functions exist for the Clebsch-Gordan and Racah coefficients for the simplest of all the unitary groups, $\mathrm{SU}(2)$. Nonetheless, a combinatorial interpretation has not yet been given, mostly because combinatoralists are unaware of these relations (developed by Regge, Schwinger, Bargmann), or, perhaps, are intimidated by their apparent complexity. Probably, it is due to lack of communication concerning the actual simplicity of the objects, concealed only by the physicists symbols for them. Combinatorial interpretations of these coefficients for SU(2) could lead to similar advances in understanding such coefficients for higher unitary groups.

(5) $3 n-j$ Coefficients. These coefficients arise when the reduction of multiple Kronecker products is considered, the real arena of many-particle physics, or when one wishes to construct irreducible representation spaces adapted to specific forms of interactions in composite physical systems. For the unitary group SU(2), the theory is 
highly developed, but incomplete [8]. Racah coefficients may be viewed as mathematical objects defined over pairs of labeled binary trees (graphs), and these, in turn, to a special class of cubic graphs. The associated problem of classifying all $3 n-j$ coefficients may be formulated as one of classifying all cubic graphs in the special class, an unsolved problem, both in mathematics and physics. Such graph theory is the playground of a whole body of combinatorics, not yet brought to bear on this important problem for physics. Much can be learned here.

(6) Excursuses. While not one of the areas in our proposal, explorations arising in relationship to planned research are vital. They occur spontaneously and unexpectedly as part of the creative process that makes advancement possible: they drive the entire process of discovery. This is an important aspect of this program of research.

\section{Importance to LANL's Science and Technology Base and National R\&D Needs}

The Los Alamos National Laboratory must maintain a capability in applied mathematics in areas extending into the realm of physics and chemistry addressed and solved in this project. Given the intersections of the collaborators' interests and motivations described here, a compelling argument for this work to be carried out at this national laboratory is the coincidence in time of topics and researchers to carry it out. This work has contributed to the general base of knowledge important for the general intellectual vitality of this nation and, in particular, of this Laboratory. Students and university lecturers come to the Laboratory because they know they can interact effectively and challengingly with its staff. Potentially, in the longer-term, some of this work can influence methodologies in the several fields to which it relates. Also, if an applied mathematics effort at the Laboratory is to be successful, it must have the respect of all its peers. The work reported here has contributed substantially to that.

\section{Scientific Approach and Results}

We summarize in this section the results obtained in each of the principal areas of the research proposed as reviewed in Section 1 under (1)-(6). The mathematical methods are typified in [17]-[20]. The publications under this proposal relevant to each topic are referenced and discussed.

(1) Structure of Representations. Publication 5 was the first to make substantive progress toward relating the irreducible unitary representations of the unitary group, as developed by physicists, to Rota's double-tableau calculus, and to make an important connection with MacMahon's Master Theorem. This was accomplished, however, for a very special class of representations. The essential idea here was the recognition that the generating function for these representations, which was already 
known to physicists, was just a variant of the one MacMahon had used for the proof of his theorem. Because the unitary irreducible representations of U(2) are quite manageable, the connection with the Rota tableau calculus could also be established for this case with the result that in the physicist's version one has to deal with nonstandard tableaux and not just the standard ones of the Rota theory. Much work is still required to understand the general case.

Progress toward bringing the unitary irreducible representations of the general unitary group $U(n)$ under the purview of combinatorics was made in a series of Conference lectures (6-9, all published or to be published), where the problem was first reformulated in terms of the ring of polynomials and then reduced to the determination of certain discrete polynomials defined over square arrays of nonnegative integers with fixed row and column sums, which are much studied, but difficult, combinatorial objects. An explicit, but very difficult recurrence relation for these coefficients was given, building uniquely the solution for $U(n)$ up to a solution for $U(n+1)$. These results clearly bring this representation theory within the domain of discrete mathematics, but these structures go well beyond anything encountered in traditional combinatorial theory, thus requiring a major effort in technique to bring then under control. But it is precisely these demanding challenges that make the theory interesting.

(2) Structure of Reduction Coefficients. Mathematicians refer to this problem as the linearization problem, since it refers to expressing a product of two functions in a certain class as a linear summation of functions in the class. The explicit determination of the discrete coefficients occurring in the linear summation together with their properties is one of the most important problems for physics as well as mathematics. In physics, these coefficients are the basis for building-up a theory of composite systems in binary fashion from the theory of a pair of constituent systems. In mathematics, these coefficients are necessary for detailed understanding of the algebra of such classes of functions. The formulation of this problem and putting it in the context of the discrete polynomials described in (1) were major parts of the lectures listed as Publications 6-9. An ancillary topic was to carry out the analogous calculations for tensor operators, which now entails generalizing the theory of irreducible polynomials in the ring of all polynomials over commuting indeterminates to a theory of irreducible tensor operators in the ring of fundamental tensor operator polynomials in which some of these basic entities are commuting and others are noncommuting. Significant results were obtained and new problems posed. 
(3) Intertwining Function. Regretfully, we found no time for this topic, partly because of dramatic progress in the next two topics, which took up our efforts. References [10]-[11] are the starting point for further developments.

(4) Generating Functions. It has become apparent in the course of research under this proposal that generating functions are a key concept for bringing comprehension to complicated physical quantities arising from symmetry considerations. Publications 6 and 8 develop some of this, for the general unitary group, but it was only when we turned to the work of Schwinger [20] that we realized the full potential of this method. We re-examined, with the help of H. W. Galbraith, consultant, Schwinger's seminal work and discovered how to generalize his methods of generating certain special coupled angular momentum state functions to the case of an arbitrary number of angular momenta. This involves beautifully the structure of continuous functions, discrete functions, and exponential differential operators, each associated with a labeled binary tree (true combinatorial objects), and a new combinatorial quantity termed the double Pfaffian, which itself relates to MacMahon's master theorem as formulated in Publication 5. The double Pfaffian is a quantity $\operatorname{det}(\mathrm{I}+\mathrm{AB})$, where $A$ and $B$ are skew-symmetric matrices. It is astonishing that mathematicians have not recognized, as Schwinger did, that this object is a perfect square! Our principal discovery was how to relate a skewsymmetric matrix to each labeled binary tree defining a coupled angular momentum state function, and in turn to formulate the generating function of a general recoupling coefficient in terms of the reciprocal of the Pfaffian. These discoveries imply many new results for the unitary group $U(n)$ as well. We reported on these preliminary results in an invited paper (Publication 11) at a recent conference in Dubna, Russia. These advances, initiated under this project, will continue development under our newly funded project: Unitary Symmetry, Discrete Mathematics, and Combinatorics.

(5) $3 \mathrm{n}-\mathrm{j}$ Coefficients. New results for the generating functions of $6-j$ and $9-j$ coefficients were given in Publication 6, and a new proof given in Publication 10 of a problem in enumerating certain cubic graphs, this insight coming from our collaborative insights. This set the stage for the developments described in (4), since $3 n-j$ coefficients are the fundamental objects that arise in the classification of all recoupling coefficients. We are optimistic that the power of the methods involving the double Pfaffian will give us new insights into the structure of the cubic graphs with which such coefficients are associated.

(6) Excursuses. The expansion of the power of an arbitrary determinant as a sum of monomials in its elements is a basic structural element in unitary group theory, and Publication 13 develops a general formula for such together with other properties. 
Ancillary problems of this sort, reflecting individual interests and insights, arise constantly in our larger program involving discrete mathematics in its larger context, which includes dynamically systems and maps of the interval, supplementing the program with details and ideas of new directions. Such is the origin of Publications 1225.

In concluding, we remark that, in our own evaluation, this project has been enormously successful in achieving its goals and in setting the course for continuing research. We attribute this to the experiences originating from different backgrounds, to a tolerance for different viewpoints, and to an environment at LANL and the Theoretical Division encouraging collaborative research.

\section{Publications}

1. Chen, W.Y.C. and Louck, J.D., "The factorial Schur function," J. Math. Phys. 34, 4144-4160 (1993).

2. Chen, W.Y.C. and Louck, J.D., "Hypergeometric Schur series," (in revision).

3. Chen, W.Y.C. and Louck, J.D., "Interpolation for symmetric functions," Adv. Math. 117, 147-156 (1996).

4. Chen, W.Y.C. and Louck, J.D., "The combinatorial power of the companion matrix," Linear Algebra and its Applications 232, 261-279 (1996).

5. Louck, J.D., "MacMahon's master theorem, double tableau, and groups." Adv. Appl. Math. 17, 143-168 (1996).

6. Chen, W.Y.C. ; Louck, J.D.; Biedenharn, L.C.; Lohe, M.A., "The role of SU(2) coefficients in SU(3)," Third SSCTP (eds. T. Lulek, W. Florek, S. Walcerz), World Scientific, Singapore, 1995, pp.150-182.

7. Louck, J.D., "Combinatorial aspects of the representations of the unitary group," Fourth SSCTP (eds. T. Lulek, W. Florek, B.Lulek, World Scientific, Singapore, 1997, pp.231-252.

8. Louck, J.D., "Unitary symmetry, combinatorics and generating functions," (to appear in Discrete Math. proceedings of June 1996, Nankai Inst. of Math., Tianjin, China. Conference on Applications of Combinatorics).

9. Louck, J.D., "Unitary symmetry, combinatorics, and special functions," XXI International Colloquium on Group Theoretical Methods in Physics, 5-20 July 1996, Goslar, Germany. Published in: Group 21: Physical Applications and Mathematical Aspects of Geometry, Groups, and Algebras, Vol. 1 (eds. H.-D. Doebner, P. Natterman, W. Scherer,) World Scientific, Singapore, 1997, pp. 50-70.

10. Chen, W.Y.C. and Louck, J.D., Enumeration of cubic graphs by inclusionexclusion, (submitted for publication, 1997). 
11. Louck, J.D.; Chen, W.Y.C.; and Galbraith, W.H., "Combinatorics of 3n-j Coefficients," (to appear in proceedings of Conference on Symmetry Methods in Physics, August 1997, Dubna, Russia).

12. Louck, J.D., "Future of the theory of angular momentum: discrete mathematics and combinatorics, " (to appear in Metropolis Memorial Volume, Birkhauser, 1997).

13. Louck, J.D., "Power of a determinant with two physical applications," (accepted for publication in Int. J. Math. and Math. Sciences).

14. Chen, W.Y.C.; Lih, K.W.; and Yeh, Y.N.,"Cyclic tableaux and Waring's formulas," Studies in Appl. Math. 94, 327-339 (1995)

15. Chen, W.Y.C. and Oliveira, J., "Implication algebra and the Metropolis-Rota axioms for cubic lattices," J. Algebra 171, 383-396 (1995).

16. Chen, W.Y.C., "Context-free grammars, differential operators and formal power series," Th. Comp. Science 117, 113-129 (1993).

17. Chen, W.Y.C. and Stanley, R., "Derangements of the n-cube," Discrete Math. 115, 65-75 (1993).

18. Chen, W.Y.C., "Induced cycle structures of the hyperoctahedral group," SIAM J. Discrete Math. 6, 353-362 (1993).

19. Louck, J.D., "Conway numbers and iteration theory."Adv. Appl. Math. 18, 181-215 (1997).

20. Beyer, W.A. and Louck, J.D., "Transfinite function iteration and surreal numbers." Adv. Appl. Math. 18, 333-350 (1997).

21. Chen, W.Y.C. and Louck, J.D., "Necklaces, MSS sequences and DNA sequences," Adv. Appl. Math. 18, 18-32 (1997).

22. Louck, J.D., "Problems in the theory of words on two letters originating from discrete dynamical systems," Annals of Comb. 1, 99-104 (1997).

23. Louck, J.D., "Doubly Stochastic matrices in quantum mechanics," (to appear in Foundations of Physics, 1997).

24. Chen, W.Y.C. and Rota, G.-C. ,"q-Hopf algebras," (submitted for publication, 1997).

25. Chen, W.Y.C. ; Li, B.Q.; and Louck, J.D., "The double Schur functions," (in preparation). 


\section{References}

[1] Louck, J.D., Theory of Angular Momentum in N-Dimensional Space. Report LA$2451,1960$.

[2] Louck, J.D., "Group theory of harmonic oscillators in n-dimensional space,"J. Math. Phys. 6, 1786-1804 (1965).

[3] Louck, J.D., "Recent progress toward a theory of tensor operators in the unitary groups,"Am. J. Phys. 38, 3-42 (1970).

[4] Louck, J.D. and Biedenharn, L.C., "A generalization of the Gauss hypergeometric series," J. Math. Anal. and Appl. 9, 423-431 (1977).

[5] Louck, J.D. and Biedenharn. L.C., "A new class of symmetric functions defined in terms of tableaux," Adv. Appl. Math. 10, 396-438 (1989).

[6] Biedenharn, L.C.; Bincer, A.M.; Lohe, M.A.; and Louck, J.D., "New relations and identities for generalized hypergeometric functions," Adv. Appl. Math. 13, 62-121 (1992).

[7] Louck, J.D. and Biedenharn, L.C., "Canonical and noncanonical methods in applications of group theory to physical problems," Adv. Quant. Chem. 23, 127-204 (1992).

[8] Louck, J.D. and Biedenharn, L.C., Angular Momentum in Quantum Physics, Vol. 8; The Racah-Wigner Algebra in Quantum Theory, Vol. 9, in Encyl. Math. and its Appl. (ed. G.-C. Rota), Cambridge University Press, Cambridge, 1981.

[9] Louck, J.D. and Biedenharn, L.C., "A pattern calculus for tensor operators in the unitary groups," Commun. Math. Phys. 8, 89-131 (1968).

[10] Louck, J.D. and Biedenharn, L.C., "Some properties of the intertwining number of the general linear group," Adv. Appl. Math. Suppl. 2, 265-311 (1986).

[11] Baclawski, K. ,"A new rule for computing Clebsch-Gordan series," Adv. Math. 5, 416-432 (1984).

[12] Macdonald, I.G., Schur functions: Themes and variations. Publ. IRMA Strasbourg, 1992,498/S-27. Actes 28 Seminare Lotharingien, pp.5-39.

[13] Golden, I. and Greene, C., "A new tableau representation for supersymmetric Schur functions," J. Algebra 170, 686-703 (1994).

[14] Golden, I. and Hamel, A.M., "Shift operators and factorial symmetric functions," J. Combin. Theory Ser. A69., 51-60 (1995).

[15] Okounkov, A. and Olshanski, O., "Shifted Schur functions," Institute for Advanced Study, preprint, Princeton, 1996.

[16] Doubilet, P., "On the foundations of combinatorial theory. VII. Symmetric functions through the theory of distributions and occupancy," Stud. Appl. Math. 51, 377-396 (1972). 
[17] Jackson, D.M. and Goulden, I.P., Combinatorial Enumeration, Wiley \& Sons, New York, 1983.

[18] Brualdi, R.A. and Ryser, H.J., Combinatorial Matrix Theory, Vol 39, in Encyl. Math. and its Appl. (ed, G.-C. Rota), Cambridge University Press, Cambridge, 1991.

[19] Turaev, V.G., Quantum Invariants of Knots and 3-manifolds, Studies in Math. 18 (de Gruyter, NY, 1994).

[20] Schwinger, J., On Angular Momentum Theory, AEC Report NYO-3071, 1952. Reprinted in: Quantum Theory of Angular Momentum (eds. L C.

Biedenharn and H. van Dam) Academic Press, NY, 1965, 229-279. 